\title{
Durchgehend geöffnet
}

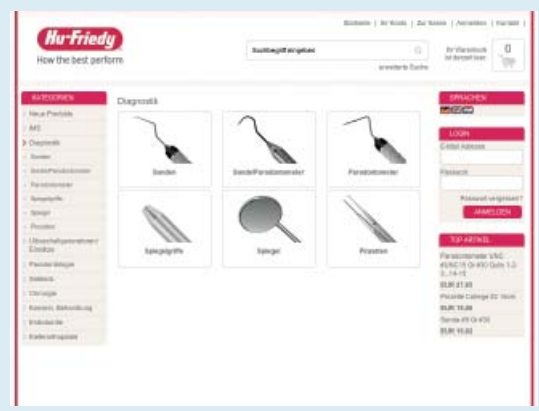

Der Dentalhersteller Hu-Friedy startet mit neuem Webshop in den Frühling. Der Einstieg nach Zielgruppen und Fachgebieten wie Chirurgie, Diagnostik, Kieferorthopädie oder Parodontologie erlaubt den di- rekten Zugriff auf das Sortiment. Flache Navigationsstrukturen und einfache Benutzerführung verkürzen den Weg zum richtigen Produkt. Für die Schlagwortrecherche gibt es ein Suchfeld. Man bekommt einen schnellen Einblick in die Produktpalette und kann die Ware per Zoomfunktion genau unter die Lupe nehmen, zusätzlich erleichtert eine detaillierte Artikelbeschreibung die Auswahl. Kunden können konkrete Preisabfragen stellen, werden vor der Bestellung ausführlich über den Bezahlvorgang informiert und erhalten eine Zusammenfassung des Auftrags- und Lieferstatus. Der Webshop steht bereits auf Deutsch, Englisch und
Holländisch zur Verfügung und ist erreichbar unter der Internetadresse http://webshop.hu-friedy.de/.

Im Rahmen der IDS präsentierte Hu-Friedy außerdem einen neuen Gesamtkatalog. Er bietet eine umfassende Illustration des Portfolios, ist aktuell auf Deutsch und Englisch erhältlich und kann über den Button auf der Seite http://deutsch.hufriedy.de als pdf heruntergeladen oder als Printexemplar angefordert werden.

Nach einer Pressemitteilung der Hu-Friedy Mfg. Co., LLC., Tuttlingen Internet: www.hu-friedy.eu 